Article

\title{
Religione, scienza e pandemia
}

\author{
GIOVANNA CAMPANI ${ }^{1}$
}

\begin{abstract}
Riassunto. Larticolo prende spunto dalle proteste di una parte consistente del mondo cattolico contro le l'interdizione delle liturgie religiose, durante il secondo "confinement" - termine francese per il lockdown -, imposto dal governo di Macron alla fine di ottobre 2020. La contestazione si è tradotta in manifestazioni davanti alle Chiese, chiedendo con forza il ripristino delle messe. La protesta si è ancora acuita quando è stata data l'autorizzazione delle messe, ma con il limite di trenta fedeli non uno di più non uno di meno - indipendentemente dalla dimensione della chiesa. Lassurdità della decisione (avallata dal Consiglio scientifico) è rivelatore del fatto che gran parte delle misure di lockdown e coprifuochi vari non sono giustificate da rigorose giustificazioni scientifiche. I cattolici inginocchiati davanti alle chiese hanno smascherato - e in modo plateale - il dogmatismo ottuso di presunte scelte ispirate dalla "scienza", di fatto dogmatiche, che paiono invertire le storiche posizioni della religione e della scienza, Le proteste dei cattolici hanno inoltre messo in luce la logica dei lockdown che impone una visione del mondo di tipo scientista che considera la pratica religiosa, con i suoi rituali, come non-essenziale per la vita dei cittadini. I cattolici che manifestano si oppongono ad una visione dell'uomo e della società secondo la quale l'unica preoccupazione è salvare la nuda vita biologica - la zoé, vita animale che s'immedesima con il ciclo naturale - con misure tecniche, ignorando la bios, che riguarda le facoltà superiori dell'uomo con i suoi bisogni spirituali.
\end{abstract}

\begin{abstract}
The article is inspired by the protests of an important part of the Catholic world against the interdiction of religious liturgies, during the second "confinement" - French term for the lockdown -, imposed by the Macron government at the end of October 2020. The protest resulted in demonstrations in front of the churches, strongly calling for the restoration of masses. The protest was further intensified when the authorization for Masses was given, but with the limit of thirty persons, not one more, not one less - independently of the size of the church. The absurdity of the decision (endorsed by the Scientific Council) reveals the fact that most of the lockdown measures and various curfews are not justified by rigorous scientific arguments. The Catholics kneeling in front of the churches have exposed - and in a blatant way - the obtuse dogmatism of alleged choices inspired by "science", which seems to reverse the historical positions of religion and science. Moreover, the logic of lockdowns imposes a scientist-like world view that considers religious practice, with its rituals, as non-essential for the life of citizens. The Catholics who demonstrate are opposed to a vision of man and society according to which the only concern is to save the bare biological life the zoé, animal life that identifies with the natural cycle -, with technical measures ignoring the bios, which concerns the higher faculties of man with his spiritual needs.
\end{abstract}

\footnotetext{
${ }^{1}$ Catedrática de Educación Intercultural y Antropología de Género en la Universidad de Florencia. Email: giovanna.campani@unifi.it
}

Copyright $\odot 2021$ The Author(s). Open Access. This in an open access article published by Firenze University Press (www.fupress.net/index.php/ccselap) and distributed under the terms of the Creative Commons Attribution 4.0 International License. The Creative Commons Public Domain Dedication waiver applies to the data made available in this article, unless otherwise stated. 
"The special mark of the modern world is not that it is skeptical, but that it is dogmatic without knowing it." G.K. Chesterton

"Never let your sense of morals prevent you from doing what is right." Salvor Hardin's epigram

Isaac Asimov

"An epidemic is a social phenomenon that has some medical aspects.".

Rudolph Virchow

\section{Introduzione}

Ho a lungo esitato prima d'accettare di scrivere un secondo articolo sul tema della pandemia da Covid-19, in un momento in cui questa è ancora in corso e ancora non si profilano i contorni della sua conclusione, al di là degli annunci mirabolanti sui vaccini, che spuntano ormai come i funghi dopo la pioggia.

Nel mio primo articolo, scritto nel mese di aprile e pubblicato nel numero 10 della rivista, ho denunciato i rischi che rappresentano le politiche di lockdown per le democrazie europee: senza sminuire la gravità della pandemia e senza dimenticare i morti per Coronavirus, ho semplicemente ricordato che, se si calpestano il diritto alla libertà personale, all'educazione ed al lavoro, in nome di un solo diritto - il diritto alla salute -, quest'ultimo, per quanto importante, finisce per diventare un diritto tiranno. In alcun momento, nella storia dell'Europa, successiva alla seconda guerra mondiale, i cittadini hanno abdicato a diritti considerati fino ad allora inalienabili, come quello alla mobilità o all'esercizio del proprio lavoro. Pur in presenza di una malattia grave, mortale in un numero limitato di casi, è legittimo chiedersi fino a che punto politiche così restrittive della libertà, nelle varie declinazioni nazionali, siano giustificate da effettivi dati scientifici.

A tutt'oggi, nonostante il proliferare degli studi, mancano analisi inconfutabili sull'insieme di misure restrittive che vanno prese per raggiungere un "livello accettabile" di ricoveri e decessi ${ }^{2}$. Allo stesso modo, mancano dati attendibili sul complesso rapporto costibenefici di simili politiche rispetto all'insieme della società - che dovrebbe essere il metro di riferimento per chi dirige - rispetto per esempio alla salute mentale, all'aumento di altre malattie non diagnosticate in tempo- come il cancro, ai danni all' economia che produrranno povertà - e quindi maggiori sofferenze, malnutrizione, degrado -, abbandono scolastico per bambini di categorie più fragili.

Infine, rimane difficile determinare il numero di vite effettivamente salvate dai lockdown -o quali restrizioni specifiche al loro interno-, sul lungo periodo, al di là di affermazioni, da parte degli stessi medici che li sostengono e, con la loro autorità, finiscono per imporli.

Ad una prima lettura - certo superficiale - dei dati mondiali, è possibile notare che i paesi che hanno fatto lockdown rigidi sono in testa alla classifica dei morti per numero

\footnotetext{
${ }^{2}$ https://blogs.mediapart.fr/carta-academica/blog/071220/covid-19-pourquoi-il-est-impossible-de-viser-unrisque-acceptable
} 
d'abitanti. Belgio, Perù, Italia, Spagna e Argentina sono i paesi in testa alle classifiche con 99 morti per 100.000 abitanti, ben al di là dei 69 della Svezia, il paese europeo che ha rifiutato il lockdown in nome della scienza epidemiologica e del rapporto stato-cittadini, ma anche degli 87 e 84 dei tanti vituperati Messico e Brasile, i cui governi si sono mossi in modo diverso, essendo impossibile per loro bloccare uneconomia con una enorme componente informale (Statista.com 30 november 2020).

Larticolo si conclude con un invito a considerare l'importanza della libertà contro la paura - della malattia e della morte - in nome di una concezione più alta e profonda della vita, che includa qualche forma di spiritualità, ed un'idea di dignità dell'esistenza, che non può essere ridotta a "nuda vita" - o "zoé", facendo riferimento alla filosofia greca, - da preservare biologicamente, opposta a "bios", che riguarda tutte le facoltà superiori dell'uomo, come sostengono i filosofi Giorgio Agamben (2020), Bernard-Bernard-Henri Lévy (2020) e Chantal Delsol (2020). Scrive quest'ultima:

Depuis le début de la période incertaine et menaçante que nous vivons, la question la plus intrigante c'est la comparaison avec les 30000 morts survenus en France en décembre 1969, frappés par la grippe de Hong-Kong, surpeuplant les hopitaux avec des détresses respiratoires, et passés complètement inaperçus. La différence avec notre réaction présente, énorme, unanime, affolée, pose question. Elle démontre une transformation majeure dans l'ordre des croyances, des références, des impératifs."

Chantal Solal individua le cause di queste differenze nella fine degli ideali occidentalicomunisti, socialisti o liberali. Gli ideali erano il motore della vita sociale e politica. Nella nostra epoca, invece, quello che predomina è l'igienismo- inteso come riorganizzazione urbana e sociale sulla base dei principi sanitari.

La seule référence qui reste après la faillite des idéologies, c'est ce que la philosophie contemporaine appelle, avec un grand nombre de variantes, la vie nue. Les gouvernements ne soccupent pratiquement plus que de protéger la vie biologique et la morale qui la sert. Aussi une épidémie devient-elle une affaire d'Etat, " une guerre », a vrai dire la seule qu'il soit important de conduire et de gagner, au detriment de tout le reste. ${ }^{3}$

In autunno, lo scenario della primavera si è ripetuto: di fronte ad un aumento dei casi di COVID-19 e di fronte alla pressione sugli ospedali per un numero alto di ricoveri, i governi europei hanno reintrodotto politiche di lockdown, che pure alcuni politici avevano promesso di non fare mai più ${ }^{4}$. Si è dunque continuato ad ignorare gli effetti devastanti di tali politiche - ormai ben studiati - sulla salute psichica delle persone, sulla violenza familiare, in particolare sulle donne, sui danni nell'apprendimento subiti dai bambini-, sul disagio dei genitori, denunciato perfino da Kate Middleton, moglie del futuro re d'Inghilterra ${ }^{5}$.

\footnotetext{
${ }^{3}$ https://www.chantaldelsol.fr/hygienisme-ou-la-vie-nue/

4 "Abbiamo visto, (...), che il lockdown è stata un'esperienza devastante, che distrugge l'economia e innalza il numero dei morti perché la gente non va più in ospedale. Dobbiamo cercare di fare di tutto per evitarlo in futuro e prendere ad esempio le popolazioni che hanno contenuto i contagi proprio con la disciplina. Oggi sono impressionato in maniera favorevole dai numeri in Italia e vorrei che il mio Paese andasse avanti così, riaprendo le scuole e le attività. Incoraggio tutti a stare attenti, lavarsi spesso le mani, rispettare le distanze, ventilare i locali, evitare i luoghi affollati, indossare la mascherina in luoghi pubblici e monitorare la situazione". http://blog.ilgiornale.it/locati/2020/08/08/il-lockdown-sono-piu-efficaci-disciplina-e-igiene/. Eppure il lockdown è stato ripetuto.

${ }^{5}$ The Covid-19 pandemic and subsequent lockdowns have caused an uptick in feelings of loneliness from parents, according to research by the Duke and Duchess of Cambridge's Royal Foundation. Some 63 per cent of parents
} 
Si è continuato a privilegiare un approccio che considera i cittadini come bambini da educare o sudditi che devono obbedire, come ha ben notato il Presidente messicano, Andres Manuel Lopez Obrador che così ha giudicato le politiche europee dei lockdown:

What it expresses is an authoritarian urge by the authorities, by the government, with all due respect, on the part of those who opt for this. (...) Curfews aren't a sign of faith in people. It's putting yourself above as authority and seeing citizens as children, like they don't understand. Not even in its worst moments did Europa have these curfews and all these measures ${ }^{6}$.

Di fronte a questo "virus che ci rende folli", titolo di un testo di Bernard-Henri Lévy (2020), non mi restava che stare in silenzio, aspettando la fine dellondata di follia ed il risveglio delle coscienze - cosa che, dopo le tragedie, la storia ci insegna - siempre sucede ${ }^{7}$.

Per quale ragione ho, alla fine, scritto quest’articolo? Perché, nel corso dell'ultimo mese, si sono prodotti alcuni eventi che s'inquadravano perfettamente nella tematica proposta dalla call del numero, Religione e Pandemia, e che sono al tempo stesso rivelatori di tendenze, svelate dalla pandemia, con le quali dobbiamo misurarci se vogliamo comprendere e forse cambiare il nostro futuro.

Durante il secondo "confinement" - termine francese per il lockdown -, imposto dal governo di Macron alla fine di ottobre 2020, che ha imposto, come in marzo, l'interdizione delle liturgie religiose, una parte - abbastanza consistente - del mondo cattolico ha contestato le misure ed ha manifestato davanti alle Chiese, chiedendo con forza il ripristino delle messe.

Un popolo di fedeli - tra cui molti giovani - inginocchiati e a mani giunte - sostenuto pure da una parte dei vescovi - offre un'immagine ben diversa da quella dei "cattivi negazionisti" o "no mask", sospettati di chissà quali legami con le destre - anche quando sventolano le bandiere arcobaleno-, regolarmente annaffiati, picchiati ed arrestati dalla polizia a Londra, Berlino o Melbourne...

Il governo, irritato dalle manifestazioni, ha cercato di fare qualche concessione, ma, volendosi mostrare magnanimi, il presidente della repubblica, il primo ministro ed il ministro della salute sono caduti nel grottesco, esasperando ancor di più il popolo di Cristo ed i suoi pastori... Prima di dare una risposta all'episcopato, il primo ministro si é, con tutta probabilità, consultato con il presidente, il ministro della salute e, probabilmente, anche se non è emerso ufficialmente, con il "Conseil scientifique", Covid-19, istituito l' 11 marzo 2020 dal Ministro della Salute Olivier Veran, "pour éclairer la décision publique dans la gestion de la situation sanitaire liée au coronavirus". Il parto di queste consultazioni è stata l'autorizzazione delle messe, ma con il limite di trenta fedeli non uno di più non uno di meno- indipendentemente dalla dimensione della chiesa. A quel

\footnotetext{
have reported feeling cut off from friends and family, up from 38 per cent before the pandemic began. There has also been a rise in the proportion of parents who feel uncomfortable seeking help, from 18 per cent feeling this way before Covid-19 outbreak to 34 per cent during.

The charity spoke to half a million people in the UK as part of its research, gathering information on what it's like to bring up children under the age of five. https://www.independent.co.uk/life-style/kate-middleton-loneliness-lockdown-parents-b1762715.html

${ }^{6}$ https://br.reuters.com/article/idUSKBN27D2SC. https://www.usnews.com/news/world/articles/2020-10-28/ mexican-president-slams-european-coronavirus-lockdown-measures.

${ }^{7}$ Titolo di una acquaforte di Francisco Goya- I disastri della guerra.
} 
punto alcune associazioni cattoliche e la stessa conferenza episcopale si sono rivolte al Conseil d'Etat, supremo organo giuridico della Repubblica, che, dichiarando la misura sproporzionata, ha obbligato il governo a ritornare sui suoi passi e a formulare una proposta meno "insensata".

I cattolici inginocchiati hanno dunque smascherato - e in modo plateale - il dogmatismo ottuso di un governo che si dichiara ispirato dalla "scienza" nelle sue scelte, rovesciando le posizioni della religione e della scienza, rispetto al semplice buonsenso, nonché alla plausibilità di dati misurabili (il distanziamento di trenta persone in $500 \mathrm{mq}$ non è paragonabile a quello che si verifica in 100). "Non è più scienza, è dogma, è il Concilio di Trento", così definisce, ironicamente, l'operato del "Conseil scientifique", l'avvocato Fabrice Di Vizio, difensore del dottor Didier Raoult, sostenitore della terapia all'idrossiclorochina, praticata in decine di paesi, ma proibita recentemente in Francia ${ }^{8}$.

Pertanto, nell'anno di grazia 2020, assistiamo ad un rovesciamento dei ruoli, quello di Galileo - che mormora tra sé e sé "Eppur si muove" - è rappresentato da un giovane inginocchiato davanti ad una Chiesa e quello del Cardinal Bellarmino dal ministro di un governo che agisce sulla base delle raccomandazioni degli esperti - "seguendo la scienza". Questo rovesciamento dei ruoli mi ha sollecitata a proporre una piccola, modesta, riflessione. Ed è nato quest'articolo...

\section{La rivolta dei cattolici francesi: Nous voulons la messe}

Il quotidiano francese Le Monde, il 16 novembre $2020^{9}$ - ha pubblicato l'immagine, toccante, di un giovane inginocchiato- una mascherina chirurgica sul viso, ma tenuta sotto il naso, che lascia scoperti dei tratti fini, i lunghi capelli raccolti, alla moda della mia giovinezza negli anni Sessanta e Settanta, le mani giunte, strette in preghiera. Intorno a lui, persone in piedi, una madre con un bebé in braccio, accanto ad una carrozzina, altri ragazzi inalberando un cartello "nous voulons la messe". Le immagini delle manifestazioni dei cattolici davanti alle Chiese di Francia hanno fatto il giro del mondo.

Se la chiusura delle Chiese durante il primo "confinement" era stata complessivamente accettata, questo non è stato il caso nel secondo "confinement" deciso il 24 ottobre 2020, per far fronte alla seconda ondata della pandemia COVID 19, che ha stabilito la sospensione di tutte le cerimonie religiose, all'eccezione dei matrimoni, limitati a sei persone e i funerali, limitati a trenta persone. La scelta di un secondo "confinement" è una scelta politica, ma è basata sul parere tecnico degli esperti-medici - scienziati del "Conseil Scientifique" - Covid-19. Le misure restrittive sono sempre discusse con i tecniciscienziati. È infatti sulla base dei pareri tecnici che "gli avvenimenti religiosi" sono stati giudicati potenziali focolai di contagio, anche in seguito ad alcuni fatti specifici. In Francia in particolare, l'incontro di 2500 fedeli di una Chiesa evangelica - La Porte Ouverte a Mulhouse nel febbraio 2020 era stato un focolaio di propagazione dell'epidemia. Aveva fatto notizia anche il caso della Chiesa Shincheonji di Jesus in Corea del Sud. Gli esperti avevano concluso che: "comme les autres rassemblements collectifs, des événements reli-

\footnotetext{
${ }^{8} \mathrm{https} / /$ likenco.fr/covid-19-pour-fabrice-di-vizio-nous-avons-un-gouvernement-anxiogene/

${ }_{9}^{9}$ https://www.lemonde.fr/societe/article/2020/11/16/des-catholiques-reclament-le-retour-des-messes-en-public_6059895_3224.html
} 
gieux sont en effet susceptibles de constituer des clusters, ce risque étant renforcé par certaines pratiques liturgiques"10.

La ricerca di un giornalista aveva portato acqua alla teoria degli incontri religiosi come "super-propagatori" dell'infezione:

Ainsi, parmi les 54 événements «super-propagateurs» (superspreader events) recensés dans 28 pays (au 23 avril 2020) par le journaliste scientifique Jonathan Kay 9 étaient liés à des activités religieuses et 5 à des funérailles. Parmi les autres cas, 19 étaient des soirées de féte, festivals, mariages ou anniversaires. On peut citer, parmi ces clusters, un temple bouddhiste à Hong Kong, les adeptes d'un gourou sikh de retour en Inde après un voyage au Canada, des rassemblements du mouvement de réveil islamique Tablighi Jamaat à Kuala Lumpur et à New Delhi, ou encore le monastère de SaintJean Bigorski.

Sebbene le pratiche liturgiche dei cattolici nulla abbiano a che vedere con quelle degli incontri delle Chiese evangeliche tra i cui membri il virus si era diffuso, fedeli ed episcopato avevano accettato le restrizioni. L'introduzione, alla fine del "confinement", di misure sanitarie rigide all'interno delle Chiese avrebbe dovuto, in teoria, evitare nuove chiusure, $\mathrm{i}$ luoghi di culto essendo almeno altrettanto sicuri che le scuole o i supermercati. Ed invece al momento del secondo "confinement", le Chiese, nonostante i banchi distanziati, il gel, gli schermi obbligatori, sono state chiuse come i cinema, i teatri, i ristoranti, e le messe proibite. I supermercati sono rimasti aperti. E la rivolta è scoppiata.

\section{Non di solo pane vive l'uomo...}

La rivolta dei cattolici è stata oggetto di analisi da parte di sociologi e politologi, che vi hanno visto l'espressione di un conflitto tra il potere religioso ed il potere politico, sia in una prospettiva liberale (le libertà fondamentali minacciate dal "totalitarismo laicista") sia in una prospettiva confessionale (il governo deve accettare la specificità del culto cattolico, che, per essere tale, ha bisogno della messa - momento fisico delleucarestia dove la comunità si ritrova ${ }^{11}$ - che non può essere ridotto ad una pratica virtuale ${ }^{12}$. Tra le varie immagini delle numerose manifestazioni, riportate dalla stampa francese, una sintetizza forse meglio di altre questo conflitto: un giovane, il volto nascosto da una maschera nera, inginocchiato davanti alla Chiesa di Saint Sulpice a Parigi, inalbera un cartello: La République n'est pas ma réligion. Rendez vous nos droits des catholiques. Rendez nous la messe. Esprimendo il rifiuto di un'identità repubblicana basata sulla laicità, il giovane si pone in continuità con le storiche battaglie susseguitesi in Francia dalla Rivoluzione in poi ${ }^{13}$. Se ad una prima lettura, questi paiono i termini del contendere, le cose sono in realtà più complicate.

\footnotetext{
${ }^{10} \mathrm{http}: / /$ www.senat.fr/fileadmin/Fichiers/Images/opecst/quatre_pages/OPECST_2020_0028_note_cultes_ covid19.pdf

${ }^{11}$ La rivendicazione della messa fa riferimento alla necessità specifica della relazione al Cristo -il culto cattolico passa per il sacrificio eucaristico. Non è possibile banalizzare il culto eucaristico- il mistero che si produce ogni volta nella messa.

12 Vedi per esempio: https://www.marianne.net/societe/laicite-et-religions/manifestations-pour-le-retablissement-des-messes-les-autorites-catholiques-ont-limpression-detre-depassees-par-leur-base-conservatrice.

${ }^{13}$ Imposizione della Costituzione civile del clero, adottata dall'Assemblea costituente nel 1790, definizione del modello identitario repubblicano, superamento dell'autorità religiosa attraverso un insegnamento laico nel corso della Terza Repubblica del 1870.
} 
Sebbene all'interno di questo movimento, vi siano delle componenti che si situano in una certa tradizione di conservatorismo cattolico, concentrare l'attenzione solo su questaspetto impedisce di cogliere le altre dimensioni, che riguardano la critica alle specifiche modalità della gestione della pandemia, come espressione di una certa concezione "antropologica" della vita e del mondo- che potremmo definire "meccanicista" che considera "essenziali" per l'uomo solo i bisogni primari di sopravvivenza fisica. Sostiene il politologo Yann Raison du Cleuziou:

La contestation de la distinction opérée par le gouvernement entre ce qui est essentiel ou non (anche sulla base delle riflessioni del "Conseil Scientifique") fait l'unanimité parmi les manifestants. Pour eux, il s'agit d'affirmer que l'identification de l'essentiel aux seules conditions matérielles d'existence, «métro, boulot, conso», est profondément révoltante. ${ }^{14}$

Rigettando una visione del mondo che considera la pratica religiosa, con i suoi rituali, come non-essenziale per la vita dei cittadini, come, evidentemente, non è essenziale la vita eterna, i manifestanti, sulla linea di Chantal Delsol, si oppongono ad una visione dell'uomo e della società secondo la quale l'unica preoccupazione è salvare la nuda vita biologica con misure tecniche definite da esperti scelti nella stragrande maggioranza tra medici, senza la presenza di alcun filosofo, per non parlare di teologi o religiosi (l'unico non -medico è unantropologa, che si è occupata essenzialmente di questioni di salute).

Perché lasciare aperti i supermercati e proibire le messe, quando nelle Chiese è possibile praticare lo stesso tipo di protocollo sanitario? Chi definisce quelle che sono "le attività essenziali" e i beni di prima necessità? Qualé la ragione sanitaria della proibizione delle messe, quando sono aperti i supermercati? Quali sono le evidenze scientifiche per questa proibizione? La proibizione del culto ha dunque manifestato un carattere ideologico, che esclude la spiritualità da quelli che sono i bisogni essenziali dell'uomo. Eppure, Gesù ha detto a Satana: "Sta scritto: Non di solo pane vivrà l'uomo, ma di ogni parola che esce dalla bocca di Dio", secondo il Vangelo di Matteo (Mt 4,1-11).

Proprio per quest'opposizione tra nuda vita - "zoé", vita animale che s'immedesima con il ciclo naturale, e "bios", che riguarda le facoltà superiori dell'uomo con i suoi bisogni spirituali, la rivolta dei cattolici di fronte al secondo "confinement" non s'iscrive nella storica opposizione del cattolicesimo alla religione de "la République" difesa con passione da l'Abbé Grégoire ${ }^{15}$. Anche la religione della République è intrisa di una forte dimensione spirituale - ed è espressione della "bios"16. Il giovane inginocchiato non è dunque un rappresentante tardivo degli Chouans di Vandea, ma il difensore di una concezione della vita che non può essere limitata alla "zoé", l’unica che le misure imposte dal governo per contenere l'epidemia COVID-19 vogliono proteggere, ma si manifesta come "bios" - ovvero la vita che l'uomo conduce nel diritto e nella libertà:

\footnotetext{
${ }^{14} \mathrm{https} / /$ www.marianne.net/societe/laicite-et-religions/manifestations-pour-le-retablissement-des-messes-les-autorites-catholiques-ont-limpression-detre-depassees-par-leur-base-conservatrice

${ }^{15}$ Almeno fosse per quello! mi viene da pensare, tra nostalgia per i principi dell'Ottantanove, e ricordo delle generose battaglie dell'Abbé Grégoire: “Grégoire, (...) rencontre dans la Révolution le signe providentiel l'autorisant à identifier, dans le même combat, l'organisation d'une église nationale "régénérée » faisant retour à la tradition égalitaire de l'eglise primitive, sans despotisme hiérarchique, au républicanisme comme théorie générale de la liberté politique et de la souveraineté du peuple. "http://classiques.uqac.ca/contemporains/boulad_ajoub_ josiane/abbe_gregoire/Abbe_Gregoire.pdf?

${ }^{16}$ La concezione dellesistenza non ridotta alla nuda vita era, infatti, parimenti condivisa dai credenti nel Cristo e dai rivoluzionari dell'Ottantanove...
} 
La dotazione spirituale - conferita alla persona dal solo fatto di essere uomo e di cui la ragione e le altre suddette facoltà superiori sono manifestazioni ed espressioni più o meno evidenti - è condizione e garanzia del bìos. Proprio perché la vita umana, pur essendo in parte zoé, la trascende e diventa bìos, acquisisce un valore assoluto che esclude ogni intervento teso ad appropriarsene e/o a manipolarla ${ }^{17}$.

\section{La surreale vicenda dei trenta fedeli}

Di fronte alle proteste davanti alle Chiese, il 26 novembre, il governo francese decide di fare una concessione e accorda l'organizzazione di messe, purché non venga superato il numero dei trenta fedeli presenti in una Chiesa: tale numero - Trenta - è fissato - é, potremmo dire, un tabù - INDIPENDENTE dalla dimensione della Chiesa che dovrebbe contenere i trenta fedeli - sia una grande cattedrale o una cappella di paese!!!!

È la goccia - o l'acquazzone - che fa traboccare il vaso. L'episcopato cattolico francese avvia un ricorso al Consiglio di Stato (Conseil d'Etat) contro il limite di trenta persone per la ripresa del culto. La decisione è presa durante una riunione di crisi, il 26 novembre stesso, che riunisce i dieci vescovi del "consiglio permanente", l'organo decisionale della Conferenza episcopale. Un comunicato stampa pubblicato venerdì mattina 27 novembre spiega questa decisione: "Nella sua dichiarazione di giovedì 26 novembre, il Primo Ministro ha annunciato il limite di 30 persone per le celebrazioni religiose da questa domenica. Questo indicatore non è né comprensibile né accettabile così comè. Di conseguenza, il Consiglio Permanente della Conferenza dei Vescovi di Francia (CEF) ha deciso di ricorrere al Consiglio di Stato questo venerdì, considerando suo dovere garantire la libertà di culto nel nostro Paese. Le numerose reazioni ricevute dai fedeli, anche di altre religioni, ci spingono ad interpellare la legge".

A Parigi, l'arcivescovo Michel Aupetit invia un messaggio ai parroci, spiegando loro la decisione in quanto il limite di trenta persone - che "manifesta un vero disprezzo per la cosa religiosa e la fede dei credenti" - è "assurdo" e "irrealizzabile". Considerando che lo "spirito evangelico" non è "mettere alla porta o escludere le persone", né "fare una selezione" tra i fedeli, il vescovo chiede "a tutti parroci a Parigi di celebrare, nel rispetto rigoroso delle distanze e dei gesti di barriera, come hanno sempre fatto. Ciascuno avrà cura di limitare il riempimento della Chiesa senza lasciare nessuno alla porta”.

Il furore dell'arcivescovo di Parigi traspare anche in una intervista a Radio Notre Dame: "Basta, devono smetterla di infantilizzarci! Fino ad ora siamo stati estremamente rispettosi di fronte alle legittime autorità come ci chiedono San Pietro e San Paolo, ma se vanno oltre e toccano le nostra coscienza e il nostro buon senso, non lo lasciamo passare". E conclude minacciando di celebrare le messe - entro il limite di un terzo dei posti disponibili secondo la proposta della Chiesa - anche se "il signor Darmanin (ministro dell'Interno) manda la polizia con i manganelli” perché i provvedimenti annunciati dal presidente, sono "totalmente stupidi" e "ridicoli". E insiste, da ex medico: "Ancora una volta, ci prendono in giro". Domenica 19 novembre il Consiglio di Stato da ragione ai vescovi ed ai fedeli che avevano fatto ricorso. "Sproporzionato", così, domenica 29 novembre, il Consiglio di Stato ha definito la decisione del governo di limitare a trenta il numero dei fedeli

\footnotetext{
${ }^{17} \mathrm{http}: / /$ www.arete-consulenzafilosofica.it/glossario.php?id=21
} 
nei luoghi di culto. Il primo ministro Jean Castex ha tre giorni di tempo per rivedere il decreto in conformità con le misure sanitarie: "I ricorrenti hanno ragione nel sostenere [...] che il suddetto divieto presenta, nello stato delle indagini e anche se rischia di essere modificato dal prossimo 15 dicembre, un carattere sproporzionato rispetto l'obiettivo di preservare la salute pubblica e costituisce quindi, in considerazione del carattere essenziale della componente in questione della libertà di culto, un attacco grave e manifestamente illegale a quest'ultima", indica il Consiglio di Stato nella sua ordinanza. "Il Presidente del Consiglio è condannato a modificare, entro tre giorni [...] le disposizioni [del decreto che limita a trenta il numero dei fedeli] adottando misure strettamente proporzionate per vigilare sui raduni e le adunanze negli istituti di culto". Così ha deciso il più alto tribunale amministrativo francese.

In tutta la vicenda, il "Conseil scientifique", che aveva consigliato il governo è rimasto silenzioso, anche se, a priori, aveva scritto, prima del "confinement" che i luoghi di culto potevano restare aperti, per non offendere troppo i fedeli (ma restare aperti non voleva dire celebrare le messe). "Les lieux de culte pourraient rester ouverts, à condition qu'ils respectent les protocoles sanitaires stricts contractualisés"18.

\section{Trenta bianchi destrier su un colle rosso?}

Il numero trenta - come limite massimo per i fedeli di un culto - sembra ossessionare i vari comitati scientifiche che, in questi tempi di pandemia, consigliano i governi. Al di là della Manica, il governo britannico dichiara apertamente di "follow the science" nella battaglia contro la pandemia.

Anche in Gran Bretagna esiste un comitato scientifico - che sembra altrettanto ossessionato che il primo ministro francese dal numero trenta -forse i trenta bianchi destrier su un colle rosso dell'indovinello di Gollum a Bilbo - riportato nell'Hobbit di Tolkien-, insensibile ai pur famosi quaranta ladroni della fiaba di Alì Baba. Il comitato ha fissato a trenta i partecipanti ai funerali, e, come in Francia, ha bloccato qualsiasi altra funzione religiosa.

Anche in Gran Bretagna i gruppi religiosi hanno protestato. Il Concilio Mussulmano britannico ha invocato un'urgente cambio nelle restrizioni. La Chiesa cattolica ha chiesto al governo di esplicitare le ragioni scientifiche della chiusura delle funzioni religiose, sostenendo che le Chiese sono sicure ed attuano tutti i protocolli sanitari. In modo pragmatico, il Cardinale Vincent Nichols e l'arcovescovo Malcolm McMahon, presidente e vice presidente dei Cattolici inglesi hanno dichiarato: "Whilst we understand the many difficult decisions facing the government, we have not yet seen any evidence whatsoever that would make the banning of communal worship, with all its human costs, a productive part of combating the virus. We ask the government to produce this evidence that justifies the cessation of acts of public worship." È stata dunque la Chiesa stessa a richiedere unevidenza scientifica che i vari comitati delle prestigiose università sono stati incapaci di fornire.

In Italia, invece, dopo la serrata dei luoghi di culto durante il primo lockdown di marzo-aprile, quando il locale comitato tecnico-scientifico aveva stabilito che le chiese erano luoghi da fuggire, in particolare per la loro peculiarità di "attirare gli anziani” che rappresentano la categoria più a "rischio epidemia", si è deciso di autorizzare le messe- anche

${ }^{18} \mathrm{https} / / /$ solidarites-sante.gouv.fr/IMG/pdf/note_conseil_scientifique_26_octobre_2020.pdf 
nelle zone rosse, a più alta circolazione del virus, - con un protocollo sanitario rinforzato. Il virus frequenta decisamente più le chiese francesi e inglesi che quelle italiane, che possono accogliere fedeli. Non va dimenticato che la Chiesa cattolica ha un grande potere in Italia, che ha esercitato in questa occasione. Ciò non toglie che il comitato tecnico scientifico italiano si sia coperto di ridicolo con le varie grida tese a bloccare la socialità umana degli italiana (i fidanzati non possono più baciarsi in pubblico) ed abbia fatto prova di inaudita crudeltà in relazione ai funerali, in assenza di dati scientifici certi sulla contagiosità dei cadaveri.

Infatti, per quello che riguarda i funerali, e, più in generale, la gestione dei defunti, il protocollo predisposto dal governo italiano (consigliato, come quello francese, da un comitato scientifico $)^{19}$ ha impedito -e tuttora impedisce- ai parenti di rivedere, anche per un solo attimo, la persona deceduta. Durante il primo lockdown anche I funerali sono stati proibiti. Le decisioni italiane sono andate ben oltre le limitazioni introdotte negli altri paesi europei. In Francia, per esempio, anche se le regole prevedevano che il cadavere fosse "immediatemente" messo in una bara e che questa fosse chiusa, la possibilità per i parenti di vedere per un momento il loro familiare, non è mai stata preclusa, in quanto "immediatamemente" non riguardava un tempo preciso. Quanto ai funerali, limitati a 20 persone, hanno potuto tenersi anche durante il primo lockdown.

In Gran Bretagna, le direttive indicano tutta una serie di precauzioni da prendere, ma insistono sull'importanza di mantenere i rituali funerari: “The grieving process and related formal and informal rituals, through which the bereaved mourn the passing of loved ones, are important for the health and wellbeing of the bereaved. Guidance on how to manage a funeral during the COVID-19 pandemic has been developed to balance the needs of the bereaved to mourn appropriately, whilst minimising the spread of COVID-19 infection."

Allo stato attuale, non esiste evidenza scientifica sulla contagiosità dei cadaveri. L'Interim Guidance dell' Organizzazione Mondiale della Sanità Infection Prevention and Control for the safe management of a dead body in the context of COVID-19 del 24 marzo 2020 stabilisce che, allo state attuale, non cé traccia di persone infettate perché esposte alle manipolazioni dei cadaveri. La letteratura scientifica sul tema è, per il momento, inesistente. Tornando a quello che pare essere un conflitto tra scienza e religione, esso rivela in realtà inquietanti dinamiche, al tempo stesso politiche e culturali, che rischiano di condurre verso mutamenti antropologici, pericolosi per la società tutt'intera, oltre che ad insopportabili limitazioni alla libertà non solo di culto ma anche di pensiero. Ma per comprendere il nostro distopico presente, dobbiamo prima fare un salto spazio-temporale verso una galassia lontana lontana...

\section{Tanto tempo fa (o tra cinquantamila anni...) in una galassia lontana lontana...}

Nel romanzo di fantascienza Foundation, Isaac Asimov narra la storia di un gruppo di scienziati, guidati dal matematico Hari Seldon, fondatore della psicostoria, una efficace

\footnotetext{
${ }^{19}$ Il modello italiano di gestione dei defunti è analogo, in una forma un po' meno brutale, a quello usato in Cina, dove, secondo China's National Health Commission, "issued regulations on February 1 stating that all victims who died of the coronavirus must be cremated at the nearest facility. No farewell ceremonies or other funeral activities involving the corpse shall be held, the NHC announcement reads".
} 
sociologia matematica - basata sulle leggi statistiche dell'azione di massa- che permette di prevedere il futuro (o almeno così paiono credere i suoi fondatori).

Avendo previsto il collasso dell'Impero galattico, Hari Selton spedisce cinquecento suoi seguaci sul lontano pianeta Terminus, con l'incarico di compilare un'enciclopedia dove sia contenuto tutto il sapere galattico, allo scopo di ridurre il tempo delle età buie, da 30.000 a 1.000 anni, fino alla nascita di un secondo impero. Sul pianeta Terminus, viene creata la Foundation - composta da scienziati ed ingeneri incaricati di preservare lo spirito della scienza e della civilta per poi costruire le basi del futuro impero galattico. "Located on the very fring of the Galactic Spiral, an only planet of an isolated sun, poor in ressources and negligible in economic value, it was never settled,until the landing of the Encyclopedists..."

Presto l'impero comincia a disgregarsi con le rivolte dei pianeti vicini di Terminus, che diventeranno i quattro regni. Gli Enciclopedisti vengono soppiantati da una dirigenza politica, che, per sopravvivvere senza diventare una colonia, inventa una sorta di religione della scienza, che viene diffusa ed imposta nei pianeti "barbari", a cui viene offerta la tecnologia, senza però la conoscenza delle basi epiistemologiche che la permettono. Un clero -giovani provenienti dai diversi pianeti e formati su Terminus, incaricato dei rituali, e del rispetto nei confronti dello Spirito Galattico, impara a lavorare con le macchine (e prima di tutto con l'energia atomica), senza essere mai in grado di riprodurla.

Il sistema religioso funziona in maniera autoritaria per alcune centinaia d'anni (con la resistenza attiva di un paio di pianeti), finché i "traders" (i mercanti) iniziano a stabilire legami commerciali con diversi pianeti, utilizzando la scienza anche per la produzione di oggetti di piacere. A quel punto si svolge lo scontro tra la casta religiosa (capeggiata dal clero di Terminus, che conosce benissimo l'impostura che sta dietro la religione della scienza, ma vuole mantenerla a tutti i costi) ed una nuova generazione di politici - meticci - che vuole legare i pianeti tra loro col pacifico commercio, mettendo al primo posto lo scambio e la politica democratica scelta dal popolo, dando sa alla scienza che alla religione il loro giusto posto nella società.

Il clero dei religiosi-scienziati che vuole la soppressione del libero pensiero e l'imposizione di leggi, regolamenti e comandamenti dogmatici, si scontra in un clamoroso processo pubblico contro il mercante che trionfa grazie all'appoggio della popolazione.

Che cosa insegnano, a noi umani, le cronache di Terminus? Prima di tutto, che le frontiere tra scienza e religione possono essere molto fragili, e che le tecniche, se insegnate nascondendone il contesto epistemologico, si traducono in uno strumento di potere, usato da una elite, che forma docili sudditi. La conoscenza scientifica in sé non ne assicura un uso "democratico", di condivisione- anzi chi è cosciente delle basi epistemologiche della scienza può essere tentato di utilizzarla per mantenere il potere, finché la casta di scienziati-sacerdoti viene rovesciata e si instaura un ordine democratico.

Le cronache di Terminus dalla lontana galassia inviano un altro messaggio importante al nostro mondo sconvolto dalla pandemia: quello che costruisce la pace tra i popoli dei diversi pianeti non è l'imposizione autoritaria di credenze religiose, anche se intrecciate con la tecnologia - imposte da un gruppo di "chierici" - gli esperti - e dunque sulla presunta razionalità scientifica.

Quello che porta la pace tra i popoli è lo scambio e l'ordine democratica: per questo alla fine sono i "traders" che prendono il potere nella Foundation, è a loro e non agli 
scienziati sacerdoti o sacerdoti scienziati che spetta il compito di attuare il passaggio dal tempo della barbarie al nuovo rinascimento di un impero. L'umanità della galassia si comporta esattamente come i terrestri: come ci insegna l'antropologia, lo scambio ha per funzione d'istituire dei rapporti umani, assicurando la comunicazione tra i membri del gruppo e di stabilire delle regole che fondano le alleanze.

L'ultimo messaggio che le cronache di Terminus possono trasmetterci è un'epigramma, attribuito a Salvor Hardin, il primo reggente del pianeta che risponde alla politica e non alla scienza: "Never let your sense of morals prevent you from doing what is right". All'epigramma ricorre il "trader" Hober Mallow, quando, stretto tra la richiesta di aiuto di un missionario della religione di Terminus (poi rivelatosi un impostore), e la necessità di entrare in comunicazione con gli abitanti del pianeta barbaro, non esita a sacrificare il missionario.

Gli scienziati che siamo costretti ad ascoltare dissertare di pandemia ad ogni ora nelle televisioni del pianeta non hanno, fino ad ora, trovato una cura valida per la malattia, ma si sono accapigliati su tutte le cure possibili, hanno collaborato alla spietata concorrenza sui vaccini che sono diventati un mercato miliardario, hanno speso soldi pubblici per fare ricerche sul rapporto tra geni Neanderthal e letalità del coronavirus e sul suono della tosse degli asintomatici...Se questa è la scienza...viene da chiedersi...Questi scienziati hanno dato al pubblico uno spettacolo talmente poco edificante che viene da chiedersi se non siano ormai più simili ai sacerdoti di Terminus, che ignorano le basi epistemologiche del loro sapere.

Come può essere possibile che i discendenti di Galileo o di Pasteur siano i personaggi che vediamo in televisione oggi? Cerchiamo di capirlo, analizzando le derive attuali della "scienza", ponendoci una domanda, che cosè, effettivamente, la Scienza?

\section{Scienza e scienziati}

In una lettera a Spencer, datata 1898, James Frazer, autore di una delle opere più lette nel corso del Novecento, il Ramo d'Oro, scrive:

Dunque, l’ordine evolutivo del pensiero umano è magia-religione-scienza. Noi, attualmente, viviamo in un'epoca di transizione tra religione e scienza, un'epoca che durera, naturalmente, per molte generazioni ancora. Sta a coloro che hanno a cuore il progresso di aiutare il trionfo finale della scienza, per quanto possono, nel loro tempo (Frazer,1986).

La visione evoluzionista - dominante nell'età vittoriana - di un passaggio benefico dalla magia alla religione - per il progresso dell'umanità - comincia ad essere messa in discussione dagli antropologi relativisti, come Franz Boas negli USA e dagli antropologi funzionalisti, come Radcliffe Brown e Malinowski, che si sforzano di dimostrare la "razionalità" dei "primitivi". Pur auspicando il trionfo del progresso scientifico, lo stesso Frazer, dopo aver consacrato la sua opera al persistere dell'irrazionale nella società contemporanea, è cosciente che la transizione dalla religione alla scienza - se mai ci sarà - durerà ancora per molte generazioni. Perché la religione è profondamente intrecciate con le modalità di costruzione delle società $e$, in fin dei conti, della civiltà umana... La religione è un elemento che fonda la nostra vita sociale, come ricordano i giovani inginocchiati davanti alle Chiese francese, la religione fonda la comunità. 
E la scienza? Che cosè la scienza? Siamo certi di parlare sempre della stessa pratica, ai tempi di Socrate e Platone, di Bruno e Galileo, di Spencer et Frazer, di Einstein e Fermi? La scienza, che nell'età dell'Umanesimo e dell'Illuminismo, si eleva a ideale contro l'oscurantismo, l'intolleranza - anche religiosa - e ogni forma di assolutismo, è la stessa pratica che, nel XIX e XX secolo offre argomenti "scientifici" al pensiero razzista? Come classificare una figura come quella di Paul Broca, eccellente studioso del cervello umano, e, al tempo stesso, difensore delle peggiori teorie razziste, poligeniste, che cercava di dimostrare col metodo sperimentale dellepoca - che i neri e i bianchi non avevano gli stessi antenati?

Probabilmente la definizione più semplice di scienza è quella di "metodo per la conoscenza", il che rende più chiaro l'aggettivo "scientifico". La scienza cerca di comprendere un fenomeno o una porzione della realtà con l'osservazione ripetibile e la misurazione oggettiva (ovvero, non si basa su impressioni, pareri, testimonianze oppure esperienze uniche o di singoli individui). L'ipotesi e la verifica sperimentale sono gli strumenti che permettono di arrivare alla previsione, la quale, in genere, permette di affermare che il fenomeno in questione è stato compreso, trasformandosi in conoscenza consolidata. Fino a quel punto, ogni teoria può essere rimessa in discussione. Come sostiene Eunoe ${ }^{20}$, sul modo di intendere e di utilizzare la scienza, nell'articolo 1, la scienza non è un catalogo di conoscenze, ma uno specifico modo di essere della conoscenza, un canone per organizzare una determinata modalità di interpretazione della realtà tramite un sistema di metafore. Pertanto, secondo l'articolo 2, La scienza non puo essere una metafisica surrogata, pertanto non è fatta di dogmi. In particolare: ogni "verita”" scientifica non è assoluta perché ha una natura probabilistica e puo essere smentita nel tempo col progredire delle conoscenze. La "scienza" è anche istituzione, gruppo sociale, l'insieme di coloro che fanno ricerca, la dirigono e di essa vivono, la cosidetta comunita scientifica, ovvero eun corpo plurale che esprime una pluralità di modelli, interpretazioni e applicazioni, spesso in reciproco conflitto, comunque irriducibili all'idea di una "verita" o di un "consenso" scientifici.

Il filosofo francese Bernard Henri Lévy ricorda che la comunità degli scienziati non è più comunitaria di altre, che è attraversata da linee di frattura, sensibilità e interessi divergenti, gelosie meschine, dispute mandarinesche e che, storicamente, molti grandi scienzati, hanno sofferto dell'accusa di stravaganza (Joseph Priestly, William Harvey, Thomas Willis, e ancora Darwin e Pasteur) (Lévy, 2020). Sempre su questo punto il manifesto sul modo di intendere e utilizzare la scienza, sostiene che le teorie e i modelli scientifici non sono semplici ipotesi o descrizioni di porzioni di mondo, ma il risultato di un vasto e intricato processo storico, culturale, economico e sociale; anche la scienza, come ogni altra attività umana, è condizionata nei suoi obiettivi e nei suoi risultati dagli interessi degli individui e dei gruppi di potere dominanti.

L'intreccio tra scienza e potere è più presente nell'ambito di alcune discipline scientifiche piuttosto che altre. La medicina è, come ci ha insegnato Michel Foucault, uno degli ambiti scientifici più compenetrato dal potere. Inoltre la medicina non è una scienza comparabile alla fisica, in quanto non vi sono leggi senza eccezioni.

In fisica e chimica sappiamo che la luce viaggia sempre alla stessa velocità o che un certo elemento solido fonde a una determinata temperatura, nella clinica accade che approssimativamente la maggior parte dei farmaci non agisce adeguatamente su una quota di pazienti vicina al $10 \%$. Inoltre, la

${ }^{20} \mathrm{http}$ ://ilpedante.org/post/eunoe-un-manifesto-per-la-scienza. 
scienza contemporanea è diventata iperspecializzata: nessuno può padroneggiare tutto ciò che serve per fare ricerca se non in un piccolo ambito; ed è prevalente anche ciò che viene chiamato "big science", ovvero un'opera collettiva in cui, pure dentro quell'ambito ristretto, ci si divide i ruoli. Articoli importanti, compresi quelli sul SARS-CoV-2 che sono stati molto discussi in queste settimane, portano la firma di decine di ricercatori, ciascuno impegnato su una piccola porzione dellesperimento (Lavazza, 2020).

Di fronte al nuovo virus, gli scienziati - in quanto esperti- sono stati chiamati ad aiutare i politici a prendere decisioni, ma è stato chiaro fin dall'inizio che non vi era accordo tra gli esperti - virologi, epidemiologi, infettivologi e clinici. Si è trattato di un fenomeno mondiale- ben descritto da un deputato inglese, nauseato dal dibattito "scientifico" sul coronavirus:

I think the Government needs to get a grip of our scientists. I'm sick and tired, and I think many people in the public are sick and tired, the science just changes. So that's fine and then we say, 'we're listening to the science', but why was the science saying something completely different beforehand? It's baffling for many people, it's causing uncertainty, it's causing worry. People don't know what the rules are any more. How can the science change from one day to the next? ${ }^{21}$.

Alla fine sono state fatte ipotesi e scelte misure i cui effetti nessuno è stato in grado di prevedere- a cominciare dagli effetti del lockdown:

Nessuno aveva mai "chiuso" interi Paesi per lunghi periodi, quindi nemmeno gli esperti potevano prevederne tutte le conseguenze, che non sono soltanto sanitarie. Qui infatti si è manifestata la prima divaricazione tra scienziati e tra scienziati e politici, con Paesi che hanno provato vie diverse, dall'Italia alla Svezia, passando per la Gran Bretagna e gli Usa, con tutte le implicazioni che abbiamo provato od osservato." (Lavazza, 2020)

Il filosofo francese Bernard Henri Lévy sostiene che, nella gestione della pandemia, si è verificata l"incestuosa unione del potere politico e medico, già denunciata da Michel Foucault, quando i medici non sono né il padreterno né gli arconti della città in preda ad un nuova pestilenza...Da qui l'esigenza, secondo Lévy, di tornare alla Repubblica, alla politica, in quanto "non si può ridurre la politica alla clinica". La salute, ha dichiarato recentemente Bernard Henri Lévy, criticando ferocemente la dittatura igienista, è qualcosa di troppo serio per essere lasciata solo ai medici o al ministro della salute. È qualcosa di globale...la salute è complicata non è solo un corpo immune di fronte ad un virus esterno...é qualcosa di olistico... 22

\section{Conclusioni}

La pandemia ci ha lasciato immagini potenti per quello che riguarda la religione: un papa che prega solo, le Chiese con i banchi bloccati, un giovane inginocchiato che chiede la messa...forse, alla fine, prevarrà questa - atto di ribellione di fronte a dogmi enunciati in nome di una scienza che non è tale, ma è espressone di un potere medico incorporato

\footnotetext{
${ }^{21} \mathrm{https}$ //www.expressandstar.com/news/uk-news/2020/08/26/tory-mp-criticises-masks-move-and-tells-ministers-to-get-a-grip-of-scientists/

${ }^{22}$ https://www.tv5monde.com/emissions/episode/l-invite-bernard-henri-levy-2
} 
nel potere politico: il giovane inginocchiato che riuscirà ad affermare che la vita è bios e non solo zoé, come già sapevano i Greci, perché non di solo pane vive l'uomo...

L'epidemia si concluderà, come tutte le epidemie- se non vorranno prolungarla per ragioni che nulla a che vedere con la scienza e nemmeno con la medicina. Lepidemia si concluderà come si sono concluse tutte le epidemie. Mentre scrivo queste parole, ho un ricordo d'infanzia: la mia città natale, mia madre, una chiesa barocca, La Chiesa del Voto, e la Cappella di Santa Cecilia, protettrice dei musicisti, elegante come una principessa, uscita da un libro di fiabe. La mamma mi racconta che quella chiesa fu eretta dopo una terribile peste che uccise la metà della popolazione della città. La peste: una malattia di cui io non ho alcuna nozione. Per me le malattie sono il morbillo, o gli orecchioni, che permettono di stare tanto tempo a casa da scuola- nel letto caldo.

La Chiesa del Voto è una versione minore - molto minore - della splendida Chiesa della Salute, che fu eretta a Venezia dopo la medesima peste del 1630. Il ritorno alla vita che queste Chiese rappresentano è il pieno ritorno ad una normalità. Dopo la morte, torna piena la vita! La Chiesa della salute non evoca una nuova normalità lugubre, fatta di volti mascherati e distanziamento sociale. La vita ha vinto la morte (collettiva non individuale, perché quella è il destino di tutto): la Chiesa della Salute sta lì a dirci che le pandemie finiscono e la bellezza della vita ritorna. Quello era il secolo XVI. Oggi, i discorsi sul post-pandemia - Niente sarà più come prima $^{23}$ - ricordano piuttosto un lugubre medioevo affascinato dal trionfo della morte - una morte che, in questo caso non è biologica - la nuda vita - zoé - dovendo essere preservata - ma sociale, culturale, artistica, religiosa la bios. È questo forse il segno, come scrive Jerome Blanchet-Gravel che la nostra civiltà sta arrivando alla sua conclusione, essendo passati da "De la bien-pensance politique à la bien-pensance hygiénique":

Refus de la mort, du tragique, de ce qui fait de la vie une aventure et non une succession de faits prévisibles et assurables, la réaction des gouvernements occidentaux est l'incarnation d'une civilisation en phase terminale. C'est au nom de la santé publique que nous affichons la mort qui nous habite. C'est au nom de la préservation de la vie que nous exhibons la dépouille de ce qui fut autrefois une civilisation d'avant-garde. Nous ne prenons même plus le temps de saluer nos disparus en inventant quelque chose d'aussi honteux et absurde que les funérailles virtuelles. " (Blanchet-Gravel, 2020).

\section{Bibliografia}

Agamben G. (2005). Il potere sovrano e la nuda vita. Homo Sacer. Torino: Einaudi.

Agamben G. (2020). A che punto siamo, Lépidemia come politica. Quodlibet.

Asimov I. (1966). Foundation. New York: Avon Books.

\footnotetext{
${ }^{23}$ «La maggior parte di noi probabilmente non ha ancora capito, e lo farà presto, che le cose non torneranno alla normalità dopo qualche settimana, o addirittura dopo qualche mese. Alcune cose non torneranno mai più», ha scritto in un articolo spiazzante quanto ben argomentato Gordon Lichfield, direttore di MIT Technology Review (il magazine della prestigiosa università americana), un'analisi dedicata ai cambiamenti nella vita personale e nel mondo del business che la pandemia, secondo la visione dello studioso, finirà per cristallizzare anche dopo che sarà attenuata .«Per fermare il coronavirus dovremo cambiare radicalmente quasi tutto quello che facciamo: come lavoriamo, facciamo esercizio fisico, socializziamo, facciamo shopping, gestiamo la nostra salute, educhiamo i nostri figli, ci prendiamo cura dei nostri familiari». La cosa è resa accettabile dal suo essere circoscritta nel tempo, ma cosa accadrebbe se diventasse qualcosa di perenne? Da Gordon Lichfield, direttore di MIT Technology Review.
} 
Blanchet-Gravel J. (2020). Quand le monde devient un «safe space». Nous rentrons en hibernation sociale. Causeur, 4 Settembre.

Delsol, C. (2020). Covid-19, on abolit l'existence à force de vouloir protéger la vie nue. In: Le Figaro, 23/10/2020

Frazer J. (1986). Il ramo d’oro. Bergamo: Euroclub.

Lavazza A. (2020). Conflitto tra scienza e politica? Solo una questione di metodo. In: Avvenire, 11 giugno 2020.

Lévy, B.H.L. (2020). Ce virus qui rend fous. Parigi: Bernard Grasset. 